\title{
Evaluation of Navier-Stokes and Euler Solutions for Leading-Edge Separation Vortices
}

K. Fujii, S. Gavali, and T. L. Holst

(AASA-TA-89458) EVALUATICN CF NAVIBR-STOKES AAD EOLER SOLUTICXS FCE LFADIAG-EDGE SEEARATICA VOEIICES (NASA) $14 \mathrm{p}$ AVail:

NTIS BC $102 / B F \quad 101$ CSCL $01 A$

N87-235E4

Unclas G3/02 0076023

June 1987

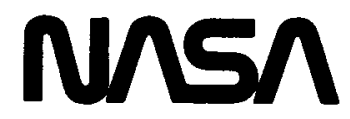

National Aeronautics and

Space Administration 


\section{Evaluation of Navier-Stokes and Euler Solutions for Leading-Edge Separation Vortices}

K. Fujii, Ames Research Center, Moffett Field, California

S. Gavali, Amdahl Corporation, Sunnyvale, California

T. L. Holst, Ames Research Center, Moffett Field, California

June 1987

\section{NRSA}

National Aeronautics and

Space Administration

Ames Research Center

Moffett Field, California 94035 


\title{
Evaluation of Navier-Stokes and Euler Solutions for Leading-Edge Separation Vortices
}

\author{
K. Fujii * \\ NASA Ames Research Center, Moffett Field, California \\ S. Gavali $\dagger$ \\ Amdahl Corporation, Sunnyvale, California \\ T.L. Holst ** \\ NASA Ames Research Center, Moffett Field, California
}

\begin{abstract}
Extensive study on the numerical simulation of the vortical flow over a double-delta wing is carried out using the "thin-layer" Navier-Stokes and Euler equations. Two important flow characteristics, vortex interaction and vortex breakdown, are successfully simulated. Grid resolution is one of the most important factors associated with the vortex problem. Computations were performed on a series of grids with various levels of refinement, coarse, medium and fine. Computations using either the coarse or medium grids fail to capture the proper physical phenomena. The computed result using a fine grid shows flow unsteadiness once the vortex breakdown takes place. The $C_{L}-\alpha$ characteristics are well predicted up to the breakdown angle of attack for all the grid distributions. The Euler solutions show fairly good agreement with the experiment on the $C_{L}-\alpha$ characteristics. However, other aspects of the solution at each angle of attack, such as the locus of the leading-edge separation vortex, are not consistent with the experiment. Even for the fine-grid Navier-Stokes computations, further grid resolution is required to obtain good quantitative agreement with the experiment.
\end{abstract}

\section{INTRODUCTION}

The vortical flow associated with leading-edge separation is believed to be essentially convection-dominated flow (in other words, rotational inviscid flow ), and thus, methods which describe flow separation and inviscid rotational flow are feasible for this purpose. Recently, many simulations of such flow fields using three-dimensional Euler and/or Navier-Stokes equations have been reported (see Ref. 1). The computed results indicate that these methods are capable of predicting a variety of vortical flow fields.

The Euler equations, which cannot describe flow separation mathematically, give us reasonable results because the flow separates at the leading edge owing to the numerical dissipation. It is claimed that these solutions are essentially independent of the choice of dissipation, at least

\footnotetext{
* NRC Research Associate

$\dagger$ Computer Performance Analyst

** Chief, Applied Computational Fluids Branch
} 
for sharp leading-edge geometries|2]. One defect of the Euler equation approach is that it cannot capture a secondary vortex near the leading edge. On the other hand, the Navier-Stokes equations have the capability to describe all the separation vortices. Most of computations have used the "thin-layer" Navier-Stokes equations; This is justified by the fact that the viscous effects are confined to a thin layer near the wall boundary and are dominated by the viscous terms associated with the strain rates normal to the wall, and the flow away from the body is essentially rotational inviscid. Viscous terms may not be negligible in the shear layer rolling up from the leading edge and in the core of the vortical flow. However, the contribution of these terms to the basic structure of the flow field is believed to be small. Besides, the viscous terms are not properly evaluated in these regions, even by the Full Navier-Stokes equations, since the computational grid is usually not fine enough. Recent computations by Fujii et al. |3| and Thomas et al. [4] showed that vortex breakdown is simulated by the "thin-layer" Navier-Stokes equations.

Although many computational results have been reported, questions remain to be answered about the reliability of the solutions. They are: 1) ls the solution quantitatively good? 2) ls the result independent of the number of the grid points to be used? 3) Is the result independent of the choice of numerical dissipation? 4) Does the Euler computation predict the vortex breakdown?

In the present paper, three-dimensional Euler and Navier-Stokes computations for the nearly incompressible vortical flow fields over a double-delta wing are presented to help to answer these questions. The flow field over a double-delta wing is more complicated than that of a delta wing. There are separation vortices from both a strake leading edge and a wing leading edge. Over the wing region, these vortices interact with each other. The inboard vortex originally induced by the strake leading edge is relatively weak in the wing region, and thus it moves outboard by the induced velocity of the wing vortex and merging of the two vortices occurs. When the angle of attack is increased, vortex breakdown occurs near the wing trailing edge. To obtain numerical solutions for leading-edge vortical flow fields, both a powerful computer and an efficient computational method are needed. Two supercomputers, the CRAY 2 at NASA Ames Research Center and the Amdahl 1200 at Amdahl Corporation, are used. The code based on the LU-ADI algorithm developed by one of the authors is appropriate for this purpose since extensive applications of this code for many other flow fields indicated reliability and efficiency.

\section{Governing Equations and Numerical Algorithm}

The partial differential equations in the generalized coordinate system, governing the three-dimensional flow of an unsteady, ideal gas can be written in conservation-law form: 


$$
\partial_{\tau} \widehat{Q}+\partial_{\xi} \widehat{E}+\partial_{\eta} \widehat{F}+\partial_{\zeta} \widehat{G}=R e^{-1} \partial_{\zeta} \widehat{S}
$$

The notations of each vector of Eq. (1) can be found in Ref. 3 . In Eq. (1), the thin-layer approximation has been introduced. Euler equations are obtained by neglecting the viscous terms in Eq. (1). The pressure, density, and velocity components are related to the energy by the following equation:

$$
p=(\gamma-1)\left(e-\rho\left(u^{2}+v^{2}+w^{2}\right) / 2\right)
$$

In the following computations, flow is assumed to be laminar and no turbulence model is used. The metrics are evaluated using second-order central-difference formulas for interior points and three-point, one-sided formulas at the boundaries. Solution of Eqs. (1) is obtained by time integration.

The numerical algorithm used here is the LU-ADI factorization method proposed by Fujii et al. [5]. Each ADI operator is decomposed into the product of lower and upper bidiagonal matrices by using a fluxvector-splitting technique and a diagonally dominant factorization. Central differencing is used in the right-hand side. In the solution process, an inversion in one direction consists of one forward sweep and one backward sweep of the scalar matrix. Thus, the LU-ADI algorithm requires little additional memory and is easily vectorized. The basic algorithm is first order in time and second order in space. For the convective terms in the right-hand side, fourth-order differencing is used except near the boundaries. Maintenance of the free stream is achieved by subtracting the free-stream fluxes from the governing equations. The artificial dissipation model developed in Ref. 5 is used in this study.

\section{RESULTS}

Most of the computations were carried out on the Amdahl 1200 supercomputer with the maximum speed of 570 MFLOPS. Two of the fine-grid Navier-Stokes solutions showing vortex breakdown were oblained on the CRAY 2 supercomputer with the maximum speed of 1700 MFLOPS for four processors. One computation was carried out on both machines and the computed $C_{P}$ distributions were within plotting accuracy. The code required $8.6 \mu \mathrm{sec}$ per grid point per iteration on the Amdahl 1200 and $20.0 \mu \mathrm{sec}$ on a single processor of the CRAY 2 .

\subsection{Grid Topology and Boundary Conditions}

The body geometry in the present study is a double-delta wing. The leading-edge swept angle is $80^{\circ}$ at the strake and $60^{\circ}$ at the wing. The thickness is $0.6 \%$ of the root chord and the leading edge is rounded. The geometry is the same as that of the experiment in Ref. 6, and is very similar to that of the experiment in Ref. 7 except for a slight difference of the wing thickness. A perspective view of the discretized region is shown in Fig. 1. The grid topology is H-type in the chordwise direction and O-type 
in the spanwise direction. The computational grid is generated by the two-dimensional, hyperbolic, grid-generation method for each chordwise station. To accurately describe the body geometry, the surface grid is carefully distributed, especially in the apex, leading-edge, and trailingedge regions.

The grid extends approximately two root chords upstream, three chords downstream, and two and one-half chords above, below, and outboard of the wing. The location of the outer boundaries is not far from the body but is probably satisfactory. The influence of the outer boundary on the solutions needs further investigation. Free-stream values are specified along the upstream and circumferential boundaries and the pressure is fixed, and the extrapolation of other physical variables are used at the outflow boundary. Bilateral symmetry is imposed to reduce the computational domain.

The grid consists of 119 points in the chordwise $(\xi)$ direction, with 14 points upstream of the body, 76 points over the body and 25 points in the wake; 101 points in the circumferential $(\eta)$ direction; and 71 points in the radial $(\zeta)$ direction. To see the effect of grid resolution, two other computational grids are used that consist of $61 \times 63 \times 41$ points in the $\xi, \eta$, and $\varsigma$ directions, respectively (medium grid), and $41 \times 33 \times 27$ points (coarse grid). It should be noted that in both the medium and the coarse grids the computational surface grids lack representation of the geometry near the apex, the trailing edge, and certain areas of the leading edge because the total number of the grid points is limited.

The computations reported in this paper utilize a Mach number of 0.3 so that comparison with a low-speed experiment can be made. The Reynolds number is $1.3 \times 10^{6}$ based on the chord length at the root corresponding to the experiment[6].

\subsection{Fine Grid Navier-Stokes Solutions}

The perspective view of the total pressure contour plots in several chordwise stations is shown in Fig. 2 at $\alpha=12^{\circ}$. Total pressure contour plots are frequently used in both experiments and computations because they are good indication of vortex patterns. At this angle of attack, two vortices, one from the strake and the other from the wing leading edge, are observed. The strength of the wing vortex increases with increasing distance downstream of the strake-wing junction. As the strength of the wing vortex increases, the weaker strake vortex is drawn outward and downward owing to the velocity field induced by the wing vortex. At the downstream station the strake vortex is observed to merge with the wing vortex. The total pressure loss due to the secondary separation vortex is also seen in the two downstream stations shown in Fig. 2. The merging process is well simulated by the computation. However, the surface pressure plots (not shown here) indicate that the computed inner strake vortex is weaker 
compared to the experiment|6|. Downstream of the strake-wing junction, the strake vortex is no longer fed by vorticity shed from the leading edge, and so remains constant in strength, or may weaken because of viscous effects. The current grid resolution and the spatial accuracy of the computational scheme are not enough to capture this process accurately. In other words, the vortex tends to lose its strength rapidly because of the numerical dissipation.

The perspective view of the total pressure contour plots at several chordwise stations is shown in Fig. 3 at $\alpha=30^{\circ}$. At this angle of attack, the merging of the strake and wing vortices occurs near the strake-wing junction. An abrupt change of the flow is observed at about $85 \%$ of chord along the streamwise direction, where the size of the vortex core suddenly becomes large. Off-surface particle-path traces shown in Fig. 4 demonstrate the breakdown of the primary vortex clearly. The flow pattern obtained in the experiment|7| for the same planform geometry is also presented. It should be noted that the experimental Reynolds number is lower and weak asymmetry of the flow is observed in the experiment. The vortex from the strake region is well-ordered and is very tightly coiled until about 80 or $85 \%$ of the chord, where an abrupt change of the flow is observed. In addition to the particles released from the strake and the wing leading edges, some particles are released from the trailing-edge region. Some of these particles move upstream to about $85 \%$ of chord and then move back downstream to the trailing-edge region. The existence of streamwise reverse flow is an evidence of the vortex breakdown. It should be noted that these reverse-flow particles continue to swirl along with the general motion of the vortex although the swirling is weak. It is also noticed that the strake vortex moves outward just behind the strake-wing junction because of the interaction with the wing vortex.

The top view of the off-surface particle path traces at $\alpha=35^{\circ}$ is shown in Fig. 5. The breakdown now takes place at about $60 \%$ chord location. The core flow from the strake leading edge has an abrupt kink there and forms a large spiral. A vertical spiral inside the breakdown region is observed at this location. This figure indicates that the core flow does not keep its original strong vorticity after the kink point. This may come from the resolution problem mentioned above. However, it is obvious that the $\alpha=35^{\circ}$ case shows a different type of breakdown from the breakdown at $\alpha=30^{\circ}$.

Careful examination of the flow field (see Ref. 3) revealed that the breakdown at $\alpha=30^{\circ}$ can be classified to be a bubble-type breakdown. Even though there is no clear evidence, the result at $\alpha=35^{\circ}$ seems to indicate the existence of the spiral-type breakdown. The structure of the vortex breakdown is not fully understood. In the experiment $[8]$ on the delta wing, for instance, it is reported that both a spiral and a bubble type of breakdown were observed to transform one to the other at random. 
The effect of turbulence is one factor always in issue. The computation assumes laminar flow, and the grid resolution is not enough to capture small eddies associated with the turbulent flow. However, global vortical flow structure in the absence of the breakdown must remain the same for both laminar and turbulent flows. Thus, the computed solutions may be useful to understand the flow structure, although the strict quantitative comparison with the experiment is very difficult.

Figure 6 shows the $C_{L}-\alpha$ curve comparison for the present NavierStokes and Euler calculations and experimental data of Ref. 6. The Navier-Stokes result shows good agreement up to about $27^{\circ}$ where vortex breakdown takes place. Careful examination of the $C_{L}-\alpha$ curve indicates nonlinear behavior of the flow field. The critical change of lift occurs at about $27^{\circ}$ angle of attack with vortex breakdown. The exact angle of attack at which vortex breakdown occurs is sensitive to the initial condition, probably because of a hysteresis effect. The $C_{L}$ value in this figure is obtained with the free-stream initial condition. When started from the solution at $\alpha=30^{\circ}$, the computed value at $\alpha=28^{\circ}$ becomes $0.912 \pm 0.005$, which is less than the value in this figure. The $C_{L}$ value at $\alpha=27^{\circ}$ becomes almost constant at the value 1.21 when started from the solution at $\alpha=25^{\circ}$, and becomes $0.966 \pm 0.004$ when started from the solution at $\alpha=28^{\circ}$. The residual does not drop enough to obtain steady-state solutions for these cases as described below although the $C_{L}$ values stay in a certain range even after 4000 to 8000 additional iterations. The outflow condition and the location of the outer boundary may explain this problem. More careful specification of the boundary conditions is necessary to discuss the quantitative feature of the solutions. It should be remembered that a similar hysteresis of the $C_{L}-\alpha$ curve is also observed in some experiments. The Euler solutions shown in Fig. 6 are discussed later.

The convergence history for the $\alpha=15^{\circ}$ case is shown in Fig. 7 in terms of the $C_{L}$ and the maximum residual. Since preliminary computation showed the flow unsteadiness at high angles of attack, the time step was set to be constant $(\Delta t=0.01)$. The computation was continued up to 5000 iterations for each case. At this angle of attack, the maximum residual drops more than three orders of magnitude within 2000 iterations and the $C_{L}$ becomes almost constant. The $C_{L}$ is not strictly constant because there is small unsteadiness in the flow field. However, the global flow field is settled and does not change. A similar tendency is obtained for every angle of attack up to the breakdown point. The computed $C_{L}$ shows clear unsteadiness above this angle of attack. However, the $C_{L}$ stays in a certain range, as is shown in Fig. 6. It should be noted that all the results for an angle of attack above $30^{\circ}$ are instantaneous rather than steady-state results that correspond to about 5000 iterations. At $\alpha=30^{\circ}$ the unsteadiness is small and the global feature of the flow does not change. At $\alpha=35^{\circ}$ flow inside the breakdown changes its feature in time. There 
may be a concern about using a diagonal-form LU-ADI solution method for unsteady computations since the algorithm is at best first-order-accurate and non-conservative in time. However, in the present study, quantitative study of the time-dependent response is not the primary interest. The main interest is associated with qualitative comparison of flow quantity amplitudes. In addition, the flow oscillations observed are slow and shockfree. The use of the diagonal form should be adequate.

\subsection{Fine-Grid Euler Solutions}

To evaluate the reliability of the Euler solution, several Euler computations were carried out under the same conditions. The code was the same as the Navier-Stokes computation, except that the viscous terms were deleted and the non-slip condition was not imposed. In addition, the same grid was used. The smoothing terms were also set to be similar.

Figure 6 indicates that Euler results showed fairly good agreement with the experiment and may be in even better agreement than the NavierStokes solution throughout the breakdown region. The perspective view of the total pressure contour plots is shown in Fig. 8 at $\alpha=12^{\circ}$. The same contour level was used as in Fig. 2. The Euler solution shows only a very weak vortical flow (thus, $C_{L}$ is lower than in the Navier-Stokes solution), and the two vortices do not merge even at the trailing edge. The result at $\alpha=30^{\circ}$ is shown in Fig. 9. Again, the contour level of Fig. 3 was used. The Euler solution now shows a clear vortical flow but fails to predict vortex breakdown. However, at $\alpha=35^{\circ}$ vortex breakdown takes place. Since solutions are sensitive to the initial condition when vortex breakdown takes place, it is difficult to draw conclusions. However, in the present case, up to the angle of attack where vortex breakdown takes place, the computed result using the Euler equations are not consistent with the experiment.

\subsection{The Grid and Smoothing Effect}

The $C_{L}-\alpha$ curves for coarse-, medium- and fine-grid solutions are shown in Fig. 10. Although the results are almost the same up to the breakdown point, only the fine-grid solution indicates the change in slope due to the vortex merging. Although not shown here, two distinct vortices are not observed anywhere at $12^{\circ}$ in the coarse- and the medium-grid solutions. The perspective views of the total pressure contour plots at $30^{\circ}$ are shown in Fig. 11 for the coarse-grid solution. The comparison of Fig. 11 and Fig. 3 indicates that the primary vortex becomes dissipative as the computational grid becomes coarse. In Fig. 3 a total pressure loss in the shear layer rolling up to the primary vortex is clearly seen. On the other hand, this is not observed in the coarse-grid solution. It is also noticed that the primary vortex is located outboard and downward when the computational grid is coarse. When the grid becomes finer, the resolution of the flow over the upper surface is improved, and the size of 
the secondary separation vortex becomes larger. This secondary vortex moves the primary vortex inboard and upward. Thus, improved resolution of the flow changes the location of the primary vortex. The $C_{L}-\alpha$ curve shows only a small discrepancy because the primary vortex is located near the surface in the coarse-grid solution, even though the vortex is weak. The total pressure loss in the center of the primary vortex core is different for each grid result. In the conical Euler solutions, the total pressure loss and the location of the primary vortex are basically indifferent to the grid size and the amount of numerical smoothing[2]. This is not true in the Navier-Stokes solutions because the resolution of the flow changes the size of the secondary separation. The effect of resolution is more serious for the double-delta wing since the strake vortex in the wing region is relatively weak. Besides, vortex breakdown occurs only in the fine-grid solution. The particle-path traces indicate no reverse flows in the medium- and coarsegrid solutions. The computed $C_{L}-\alpha$ curve shows a good result, even for the coarse grid computation. Thus, coarse grid may be useful for the engineering purposes. However, it should be remembered that the solution may lack flow structure.

To check the effect of the numerical smoothing terms, the coefficient of the smoothing terms was artificially increased to be three times larger. The result still displayed the existence of vortex breakdown and was close to the result in Fig. 3, except that the secondary separation vortex was smaller. This, along with the Euler solution in Fig. 9, indicates that the smoothing terms are not a key parameter in deciding flow-field structure. The viscous shear layers over the surface appear to be the most important factor in deciding the vortical flow structure.

\section{SUMMARY AND CONCLUSIONS}

Extensive study on the numerical simulation of the vortical flow over a double-delta wing was carried out using the "thin-layer" Navier-Stokes and Euler equations. Two important flow characteristics, vortex interaction and vortex breakdown, have been successfully simulated. The $C_{L}-$ $\alpha$ characteristics were well predicted up to the breakdown angle of attack. The Euler solutions showed fairly good agreement with the experiment on the $C_{L}-\alpha$ characteristics. However, other aspects of the solution at each angle of attack such as the locus of the leading-edge separation vortex were not consistent with the experiment. Even for the Navier-Stokes computations using a fine grid, further grid resolution is required to obtain good quantitative agreement with the experiment. Additional research into the careful specification of boundary conditions may also be required.

\section{ACKNOWLEDGEMENT}

Conducting a parametric study using a large number of grid points requires extensive cpu time. The authors would like to express their gratitude to the Amdahl Corp. in allowing them to use the VPS system. 


\section{REFERENCES}

1 NEWSOME, R. W. AND KANIIL, O. A. - Vortical Flow Dynamics - Physical Aspects and Numerical Simulation. AIAA Paper 87-0205, Jan. 1987.

2 POWELl, K., MURMAN. E. M., PEREZ, E. and BARON, J. Total Pressure Loss in Vortical Solutions of the Conical Euler Equations. AlAA Paper 85-1701, July 1985.

${ }^{3}$ FUJII, K. and SCHIFF, L.B. - Numerical Simulation of Vortical Flows Over a Strake-Delta Wing. AIAA Paper 87-1229, June 1987.

4 THOMAS, J. L., TAYLOR, S. L. and ANDERSON, W. K. - NavierStokes Computations of Vortical Flows Over Low Aspect Ratio Wings. AIAA Paper 87-0207, Jan. 1987.

${ }^{5}$ FUJII, K. and OBAYASHII, S. - Navier-Stokes Simulation of Transonic Flow over Wing-Fuselage Combinations. AlAA Paper 861831 , June 1986.

6 BRENNENSTUHL, U and HUMMEL, D. - Vortex Formation over Double-Delta Wings. ICAS Paper 82-6.6.3, The proc. 13th Cong. Intern. Coun. Aeronaut. Sci. August 1982.

7 THOMPSON, D. H. - A Visualization Study of the Vortex Flow around Double-Delta Wings. ABL-AERO-R-165, Dept. Defence Australia, August 1985.

8 PAYNE, F. M., NG, T. T., NELSON, R. C. and SCHIFF, L. B. - Visualization and Flow Surveys of the Leading Edge Vortex Structure on Delta Wing Planforms. AIA A Paper 86-0330, Jan. 1987.

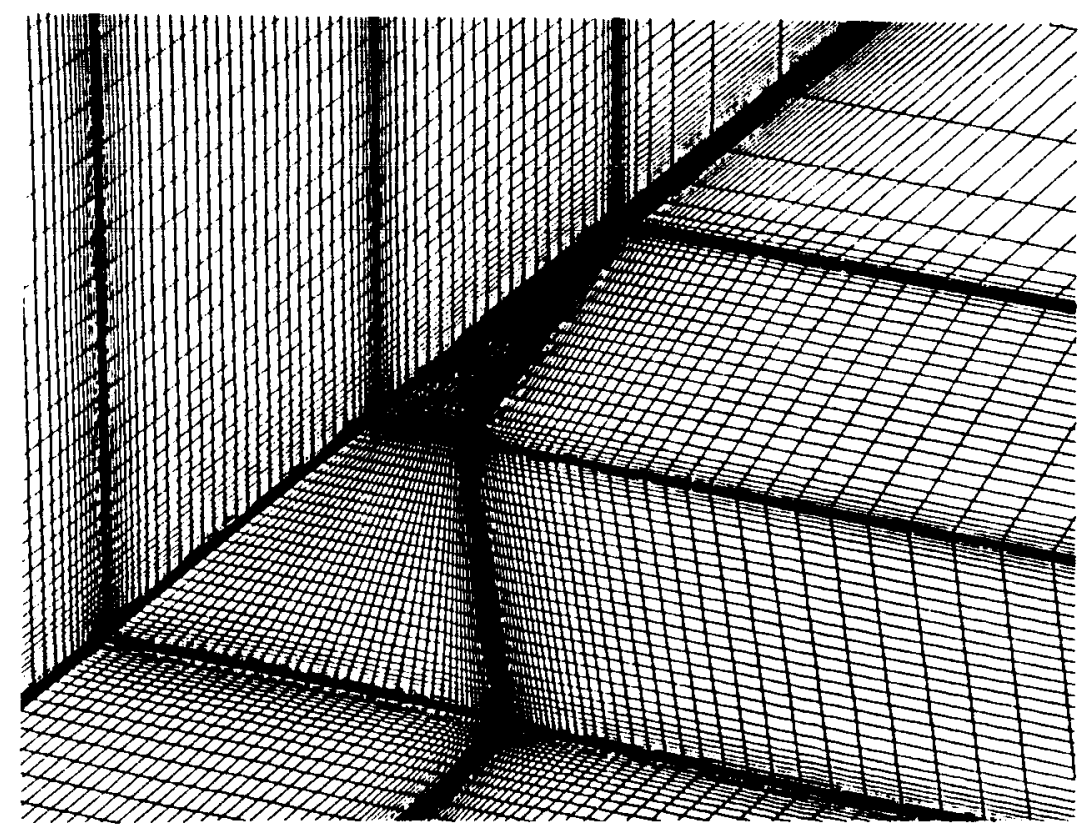

Fig. 1 Close-up view of the discretized region. 


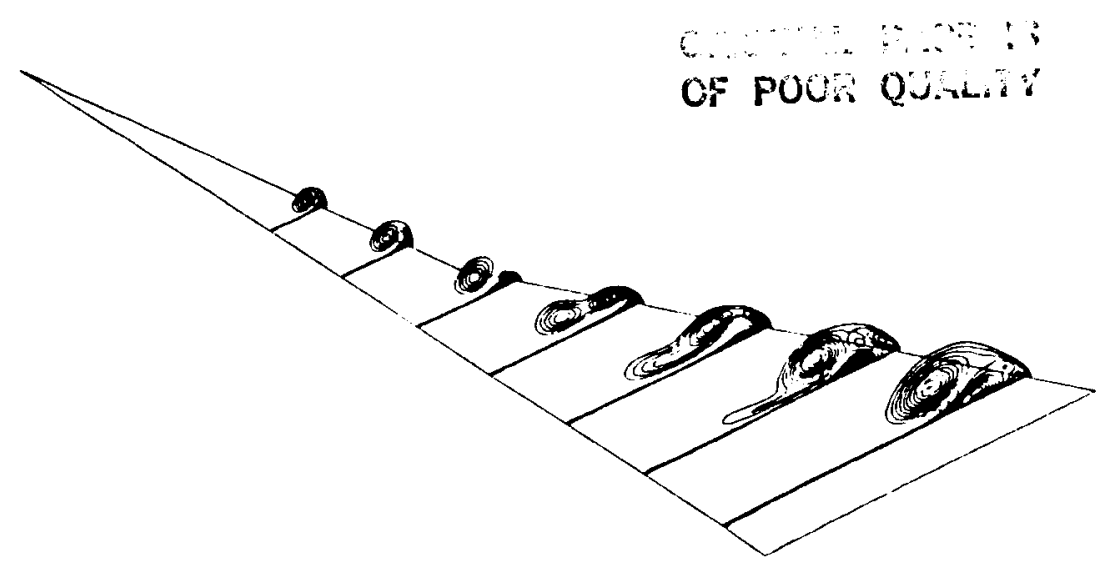

Fig. 2 Perspective view of the spanwise total pressure contour plots; $M_{\infty}=0.3, \operatorname{Re}=1.3 \times 10^{\circ}, \alpha=12.0^{\circ}$.

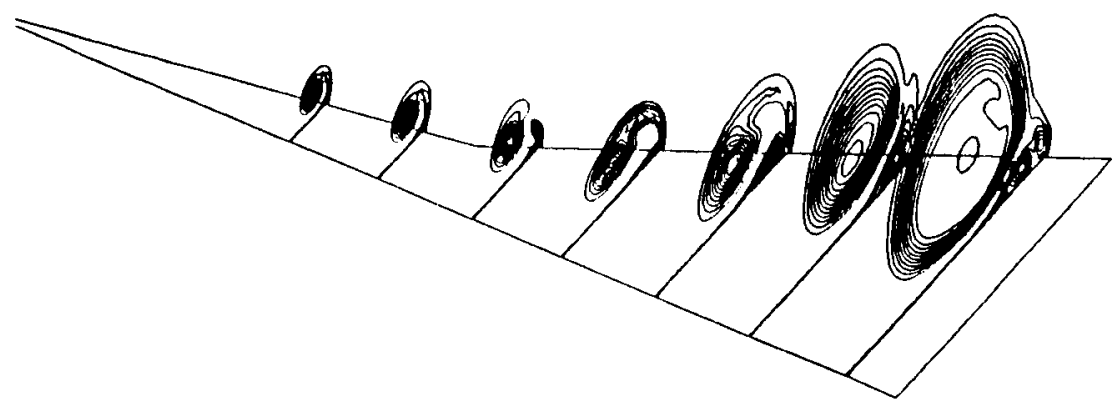

Fig. 3 Perspective view of the spanwise total pressure contour plots; $M_{\infty}=0.3, \operatorname{Re}=1.3 \times 10^{\complement}, \alpha=30.0^{\circ}$.

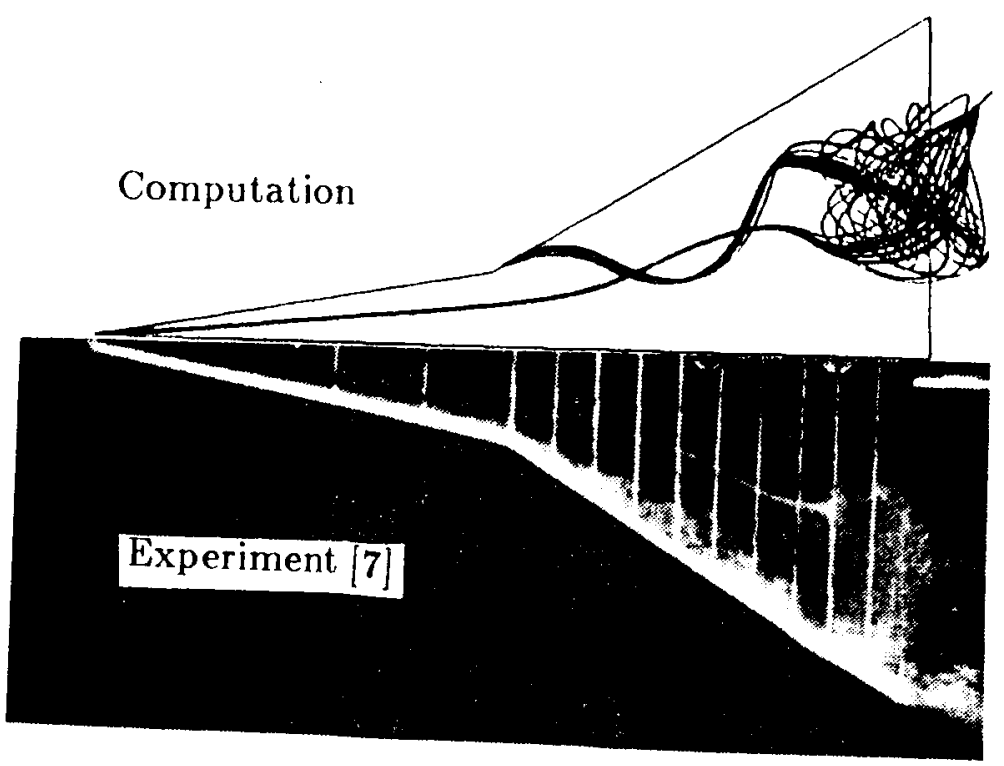

Fig. 4 Computed off-surface particle-path pattern compared with the experiment 77 ; $M_{\infty}=0.3, \alpha=30.0^{\circ}, \operatorname{Re}=1.3 \times 10^{\circ}$ (Incompressible and $\operatorname{Re}=1.0 \times 10^{5}$ for the experiment). 


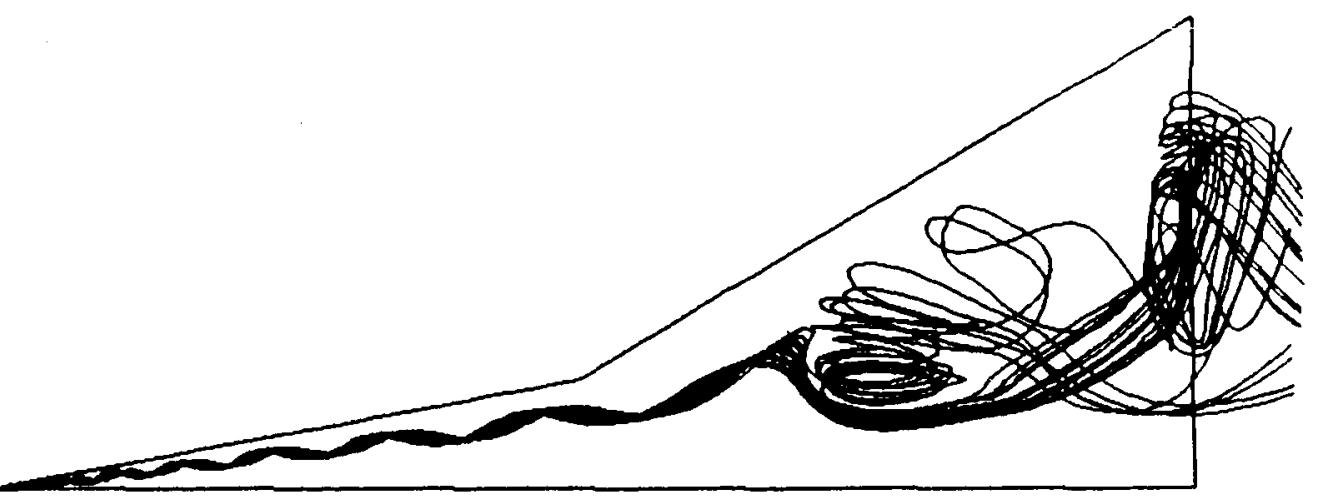

Fig. 5 Computed off-surface particle-path pattern; $M_{\infty}=0.3, \operatorname{Re}=1.3 \times 10^{6}$, $\alpha=35.0^{\circ}$.

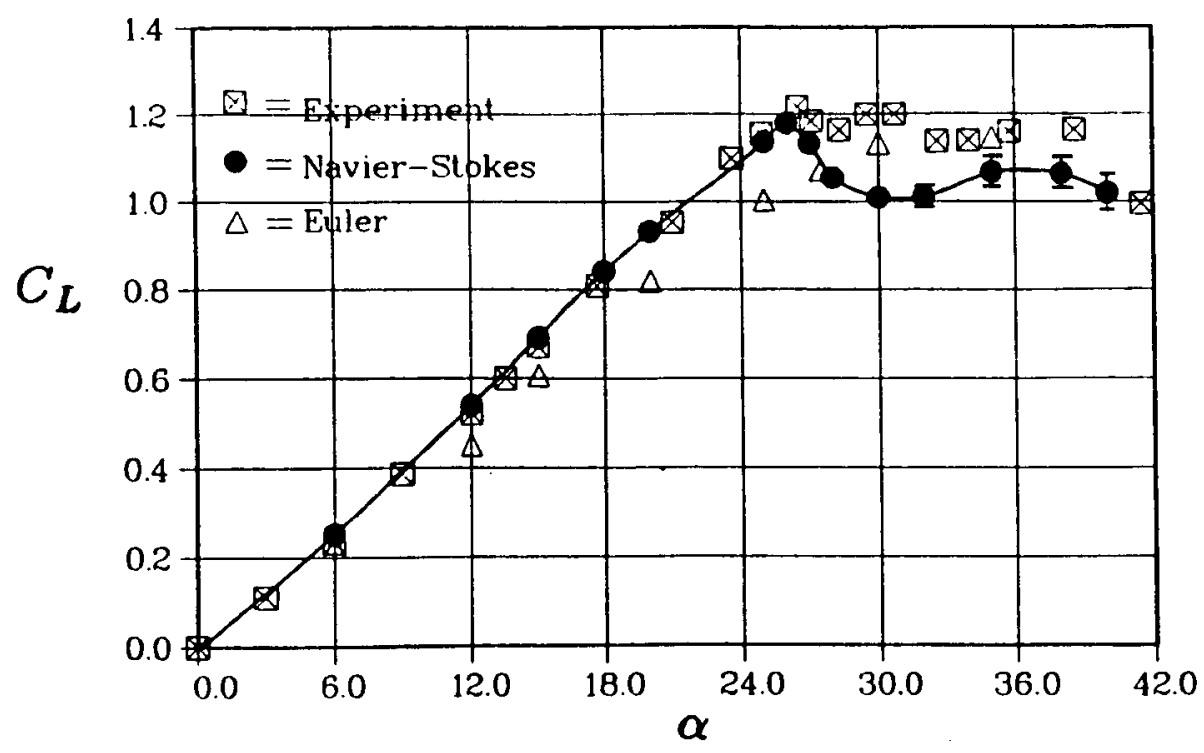

Fig. $6 C_{L}$.vs. $\alpha$ characteristics; $M_{\infty}=0.3, \mathrm{Re}=1.3 \times 10^{6}$.

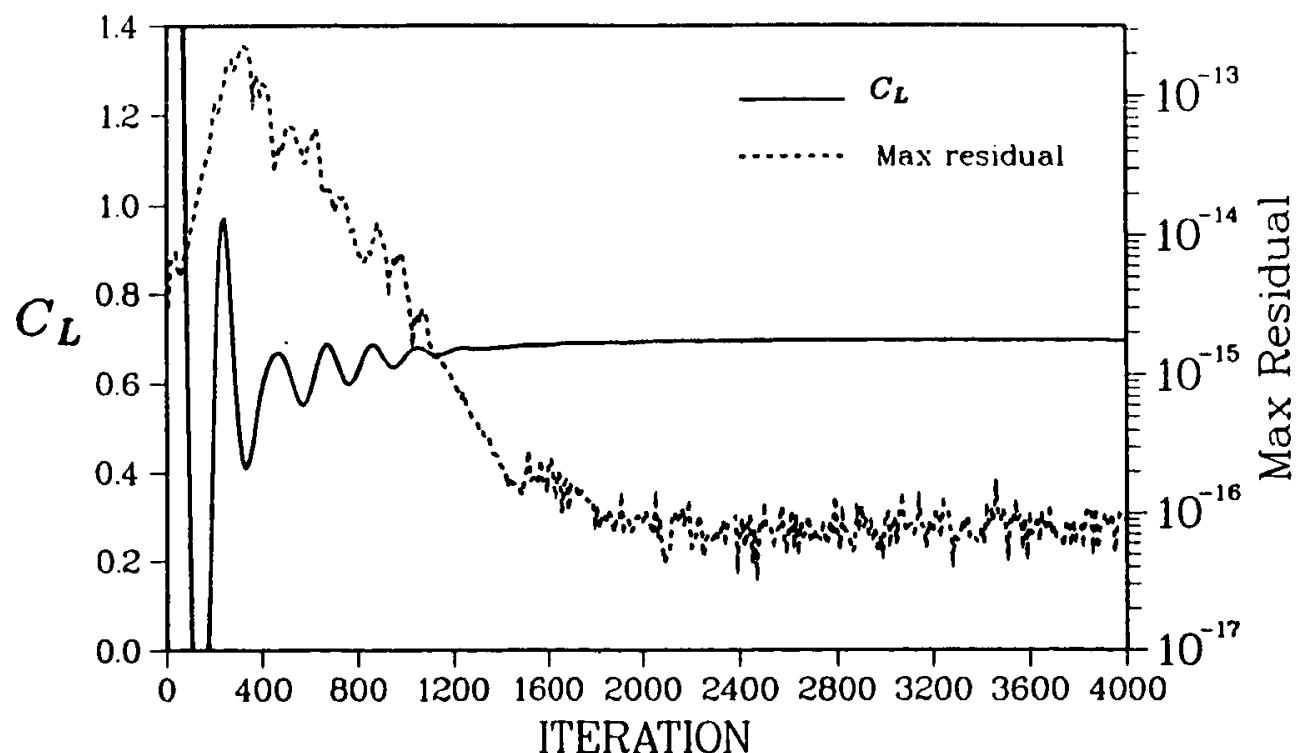

Fig. $7 C_{L}$ and maximum residual history for $\alpha=15.0^{\circ} ; M_{\infty}=0.3$, $\operatorname{Re}=1.3 \times 10^{6}$. 


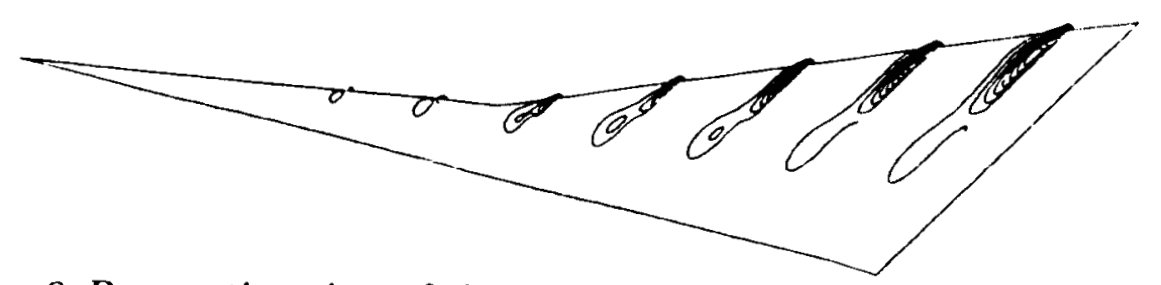

Fig. 8 Perspective view of the spanwise total pressure contour plots (Luler solution); $M_{\infty}=0.3, \alpha=12.0^{\circ}$.

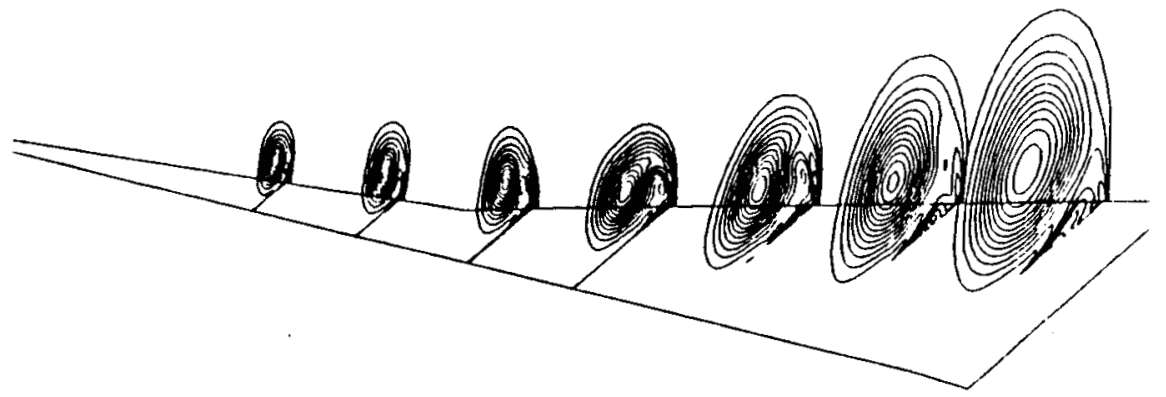

Fig. 9 l'erspective view of the spanwise total pressure contour plots (Euler solution); $M_{\infty}=0.3, \alpha=30.0^{\circ}$.

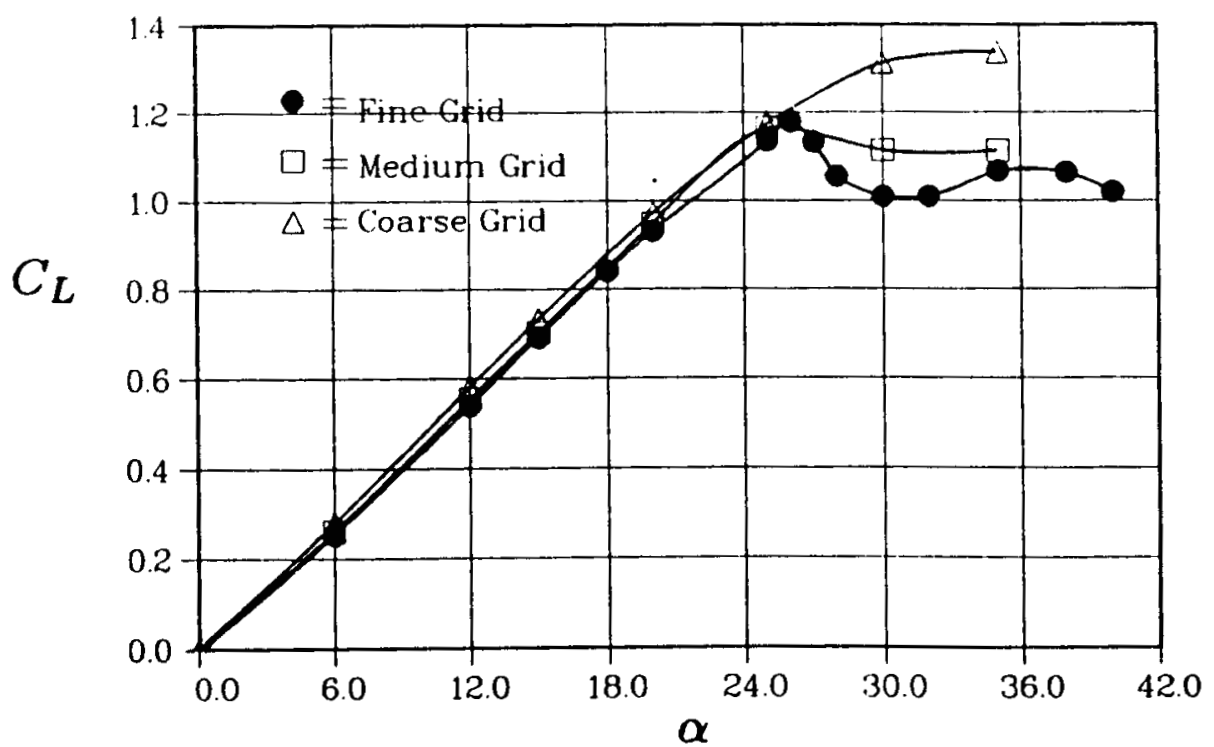

Fig. $10 C_{L}$.vs. $\alpha$ characteristics (grid effect); $M_{\infty}=0.3, \operatorname{Re}=1.3 \times 10^{6}$.

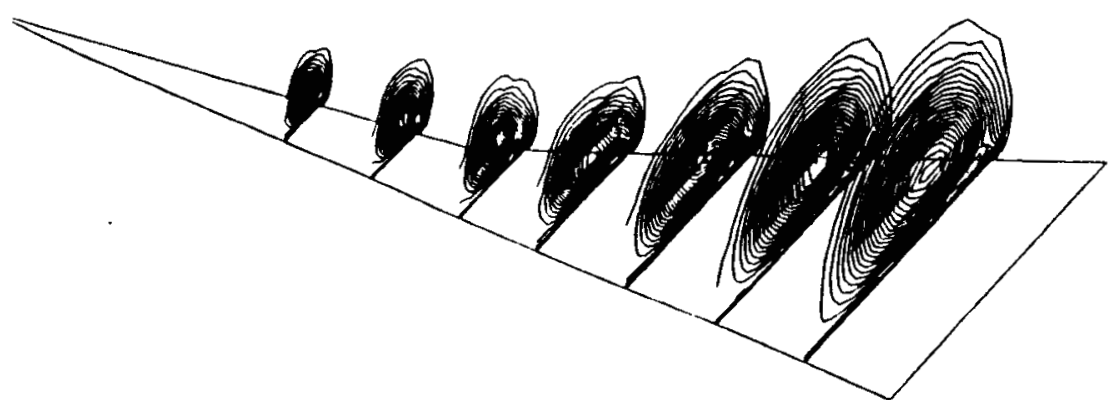

Fig. 11 Perspective view of the spanwise total pressure contour plots (coarse grid solution); $M_{\infty}=0.3, \operatorname{Re}=1.3 \times 10^{6}, \alpha=30.0^{\circ}$. 


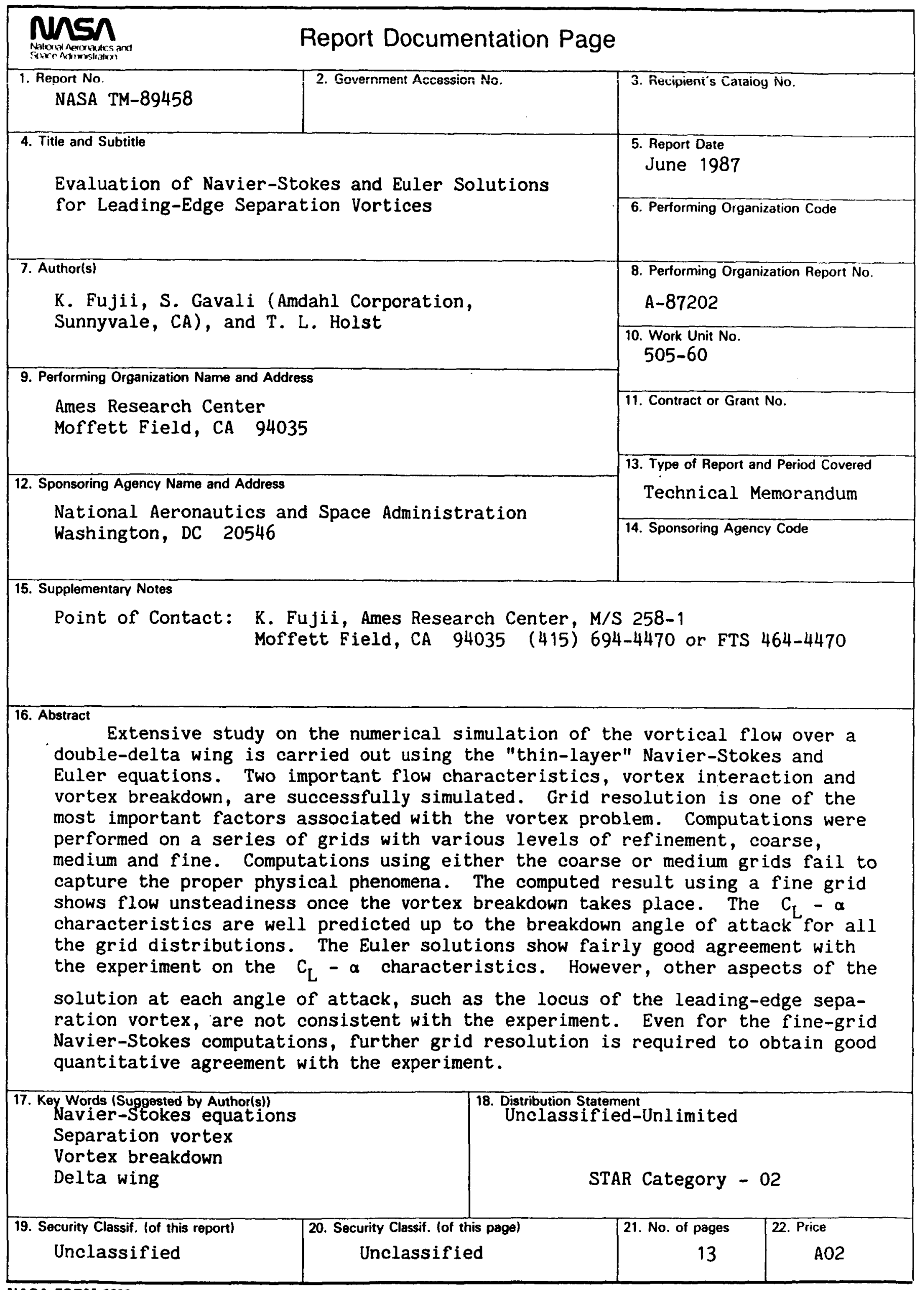

